\title{
The Risk-Sharing Problem
}

\author{
by Denis Moffet *
}

\section{Introduction}

The purpose of this paper is to pursue the works of Karl Borch and Kenneth Arrow on the risk-sharing problem. It will be seen how a classical result due to Arrow can be obtained directly from a more general theorem proved by Borch. The implications of Borch's theorem on the negotiation of an arrangement between an insured and an insurer will also be illustrated.

\section{Borch's theorem}

Let $n$ denote the number of individuals facing a risky situation the elements of which being

$W_{i}$ : the wealth of individual $i$

$X_{i} \quad$ : a random variable representing the loss to individual $i, 0 \leqslant X_{i} \leqslant W_{i}$ $F_{i}\left(x_{i}\right)$ : distribution function.

The random variables $X_{1}, X_{2}, \ldots, X_{n}$ are supposed to be stochastically independent.

It is supposed that each individual's preferences can be represented by a utility function $u_{i}(\cdot)$ having the following characteristics :

$$
\begin{aligned}
& u_{i}^{\prime}(\cdot)>0 \text { i.e. positive marginal utility } \\
& u_{i}^{\prime \prime}(\cdot)<0 \text { i.e. decreasing marginal utility, }
\end{aligned}
$$

which is equivalent to saying that each individual is risk adverse. Furthermore it will be assumed that each individual aims at maximizing the expected utility of his wealth.

In the initial state of uncertainty, the expected utility of individual $i$ is

$$
U_{i} \equiv \int_{0}^{W_{i}} u_{i}\left(W_{i}-x_{i}\right) d F_{i}\left(x_{i}\right)
$$

The objective of the $n$ individuals is to redistribute among themselves the losses they can incur according to a mechanism acceptable by all.

* The author is associate professor of actuarial science at Laval University in Quebec City. He is grateful to Philippe Capérad and Graham Lord for helpful comments. 
Let $y_{i}\left(x_{1}, x_{2}, \ldots, x_{n}\right)$ denote the amount individual $i$ will have to support if the losses effectively incurred are $x_{1}, x_{2}, \ldots, x_{n}$ for each individual respectively. ${ }^{1}$ Leaving aside transaction costs, the conservation equation is then

$$
\sum_{i=1}^{n} y_{i}(x) \equiv \sum_{i=1}^{n} x_{i}
$$

For individual $i$, the expected utility given by such an arrangement is

$$
U_{i}(y) \equiv \int_{0}^{W_{i}} \ldots \int_{0}^{W} u_{i}\left[W_{i}-y_{i}(x)\right] d F_{1}\left(x_{1}\right) \ldots d F_{n}\left(x_{n}\right) .
$$

The necessary condition for this arrangement to be acceptable by all is

$$
U_{i}(y) \geqslant U_{i}, \quad i=1,2, \ldots, n
$$

If the $n$ individuals behave rationally, they will not accept the arrangement $\left\{y_{1}, y_{2}, \ldots, y_{n}\right\}$ if there exists another one $\left\{\bar{y}_{1}, \bar{y}_{2}, \ldots, \bar{y}_{n}\right\}$ such that

$$
U_{i}(\bar{y}) \geqslant U_{i}(y), \quad i=1,2, \ldots, n
$$

where (1) holds with a strict unequality for at least one individual. If it is impossible to find an arrangement $\left\{\bar{y}_{1}, \bar{y}_{2}, \ldots, \bar{y}_{n}\right\}$ satisfying condition (1), then the arrangement $\left\{y_{1}, y_{2}, \ldots, y_{n}\right\}$ is Pareto optimal. ${ }^{2}$

Borch's theorem [2] which was proved in 1960 can now be stated as follows :

If $u_{i}^{\prime}(\cdot)>0$ and $u_{i}^{\prime \prime}(\cdot)<0$, for $i=1,2, \ldots, n$, then an arrangement $\left\{y_{i}, y_{2}, \ldots, y_{n}\right\}$ is Pareto optimal if and only if

$$
u_{i}^{\prime}\left[W_{i}-y_{i}(x)\right]=k_{i} u_{1}^{\prime}\left[W_{1}-y_{1}(x)\right]
$$

where $k_{2}, k_{3}, \ldots, k_{n}$ are positive constants chosen arbitrarily and $k_{1}=1$.

The implications of this theorem are not obvious. However Borch has shown as a corollary that

$$
y_{i}(x)=\Phi_{i}\left(\sum_{i=1}^{n} x_{i}\right), \quad i=1,2, \ldots, n
$$

if the arrangement $\left\{y_{1}, y_{2}, \ldots, y_{n}\right\}$ is Pareto optimal; which means that, in order to reach an optimal arrangement, a pool must be formed. In the case where the individuals are insurance companies, a description of the situation is offered by Borch himself :

"The fact that $y_{i}(x)$ is a function of $\sum_{i}^{n} x_{i}$ alone means that the only Pareto optimal arrangement is that the companies cede their entire portfolio to a pool, and then agree on some rule as to how payment of claims against the pool should be divided among

1 For simplicity, let $x$ denote the vector $\left(x_{1}, x_{2}, \ldots, x_{n}\right)$.

2 In honour of the economist Vilfredo Pareto (1848-1923). 
the companies. There may be an infinity of such rules, since the positive constants can be chosen arbitrarily."

In practice a means must be found to reduce as much as possible the domain of arrangements which are acceptable in the sense of Borch's theorem. In the next section a special risk-sharing problem will be studied and it will then be seen how the arbitrariness can be greatly reduced.

\section{The search for an optimal insurance scheme}

Let us consider a case involving two individuals : individual 1 is a potential insured and individual 2 is an insurer. In the initial situation, only the individual 1 can incur a loss, i.e. $x_{2}=0$ with probability equal to one. Let us now consider an arrangement according to which the insurer is bound, after having been paid a premium $P$, to pay an indemnity $I(x)$ to individual 1 should the latter incur a loss amounting to $x$.

Let us suppose that the initial wealth of these individuals is $W_{1}$ and $W_{2}$ respectively ; the insurance scheme will be Pareto optimal if and only if

$$
u_{2}^{\prime}\left[W_{2}+P-I(x)\right]=\mathrm{k} u_{1}^{\prime}\left[W_{1}-P-x+I(x)\right]
$$

Equation (2) is a straighforward application of Borch's theorem where

$$
\begin{array}{ll}
y_{1}(x) \equiv P+x-I(x), & x_{1} \equiv x \\
y_{2}(x) \equiv I(x)-P \quad, & x_{2} \equiv 0 .
\end{array}
$$

As it stands, equation (2) does not reveal much, it is necessary to reduce the set of feasible solutions. In practice an indemnity is not paid out unless a loss has been incurred. This implies the following initial condition

$$
I(0)=0
$$

The fundamental equation (2) must be verified when the loss is equal to zero, therefore it follows from (2) and (3) that

$$
k=u_{2}^{\prime}\left[W_{2}+P\right] / u_{1}^{\prime}\left[W_{1}-P\right]
$$

Since $u_{i}^{\prime}(\cdot)>0$ and $u_{i}^{\prime \prime}(\cdot)<0$, it follows from (4) that there is a one-to-one correspondence between $k$ and $P$.

Substituting in (2) the expression for $k$ obtained in (4) yields

$$
\frac{u_{2}^{\prime}\left[W_{2}+P-I(x)\right]}{u_{2}^{\prime}\left[W_{2}+P\right]}=\frac{u_{1}{ }^{\prime}\left[W_{1}-P-x+I(x)\right]}{u_{1}^{\prime}\left[W_{1}-P\right]}
$$

Differentiating in (5) with respect to $x$ one obtains

$$
\frac{\partial I(x)}{\partial x}=\frac{u_{2}{ }^{\prime}\left[W_{1}+P\right] u_{1}{ }^{\prime \prime}[W-P-x+I(x)]}{\lambda}
$$

where $\lambda=u_{1}^{\prime}\left[W_{1}-P\right] u_{2}{ }^{\prime}\left[W_{2}+P-I(x)\right]+u_{2}^{\prime}\left[W_{2}+P\right] u_{1}{ }^{\prime \prime}\left[W_{1}-P-x+I(x)\right]$. Since $u_{i}^{\prime}(\cdot)>0$ and $u_{i}^{\prime \prime}(\cdot)<0$, it follows that 


$$
0<\frac{\partial I(x)}{\partial x}<1
$$

Since from (3), $I(0)=0$, it follows according to the mean-value theorem that

$$
0<I(x)<x \quad \text { for } x>0
$$

The result expressed by (6) indicates that coinsurance is the optimal insurance scheme if both the insured and the insurer are risk averse. This is a classical result of the economic theory of risk which has been proved in a different way in 1963 by Arrow [1]. The proof is somewhat simpler here and furthermore illustrates the depth of Borch's theorem.

In every day life, one observes that coinsurance is not always the insurance scheme which is adopted. In motor vehicle insurance for example, a scheme with a deductible is generally used. Under such an arrangement a level $D$ is chosen : if $x \leqslant D$ then $I(x)=0$ and if $x>D$ then $I(x)=x-D$. As a result

and

$$
\frac{\partial I(x)}{\partial x}=0 \text { for } x<D
$$

$$
\frac{\partial I(x)}{\partial x}=1 \text { for } x>D .
$$

According to the theory which has just been developed, the insurance with a deductible cannot be Pareto optimal if both the insured and the insurer are risk averse. Since it can be postulated that it is in fact the prevailing situation, one must wonder wether people are irrational or if it is the theory which is deficient.

It can be hazardous to bear judgment on people's rationality. A weakness of the theory can however be pointed out: namely the absence of transaction costs. In practice, small losses should not be insured since settlement expenses can possibly amount to more than the losses themselves, therefore a deductible makes sense. However, one can still ask himself why coinsurance is not adopted for losses exceeding the level $D$, it could perhaps help to reduce claims since the insured would then have an immediate motivation in preventing losses. These considerations are not without interest but pursuing them further would lead us outside the scope of this study.

Since both individuals are risk averse, equation (5) shows that any pair $(P, x)$ determines a unique value for $I(x)$. In Appendix I, it is shown that $I(x)$ can be approximated by a linear function of $x$, which turns out to be

$$
I(x) \approx \frac{R_{A 1}\left(W_{1}-P\right)}{R_{A 1}\left(W_{1}-P\right)+R_{A 2}\left(W_{2}+P\right)} x
$$

where $R_{A i}(\cdot)$ is the Arrow-Pratt measure of absolute risk aversion defined as

$$
R_{A i}(\cdot) \equiv-\frac{u_{i}^{\prime \prime}(\cdot)}{u_{i}^{\prime}(\cdot)}
$$

The closed form approximation for $I(x)$ expressed by (8) is quite interesting. Indeed, one can see at a glance how each relevant variable contributes to the determination of 
the optimal indemnity. It is of no surprise to see that the risk aversion of both the insured and the insurer influences also the optimal indemnity.

The problem boils down to the selection of a scheme acceptable by both parties. By considering a simple example, it will be shown in the next section how this problem can be solved.

\section{A numerical example}

Individual 1 owning an initial wealth $W_{1}=40$ can suffer a loss amounting to $x$ according to the probability law described in Table 1. An insurer owning an initial wealth $W_{2}=100$ is willing to negociate a risk-sharing arrangement with individual 1 . For the purpose of this example, let us suppose that the utility function of each of these individuals is respectively

$$
u_{1}(X)=X^{\alpha} \quad \text { and } \quad u_{2}(X)=X^{\beta}
$$

with $0<\alpha<1$ and $0<\beta<1$.

Table 1 - The probability law governing the loss $x$

\begin{tabular}{|c|c|r|r|r|r|r|r|r|r|r|}
\hline$x$ & 0 & 1 & 2 & 3 & 4 & 5 & 7 & 10 & 15 & 20 \\
\hline prob & .30 & .05 & .06 & .08 & .10 & .13 & .15 & .07 & .04 & .02 \\
\hline
\end{tabular}
becomes

For the problem under consideration the fundamental equation given by (5)

$$
[100+P]^{\beta-1}[40-P-x-I(x)]^{\alpha-1}=[40-P]^{\alpha-1}[100+P-I(x)]^{\beta-1}
$$

From this latter equation it is possible to derive the optimal indemnity $I(x)$ for any triple $(P, \alpha, \beta)$. In Table II some indemnity schemes have been calculated.

Table II - Optimal indemnity $I(x)$ according to $(P, \alpha, \beta)$

\begin{tabular}{|c|c|c|c|c|c|c|}
\cline { 2 - 7 } \multicolumn{1}{c|}{} & \multicolumn{3}{c|}{$\alpha=1 / 4, \beta=1 / 2$} & \multicolumn{3}{c|}{$\alpha=1 / 8, \beta=1 / 2$} \\
\hline$x$ & $P=1$ & $P=5$ & $P=10$ & $P=1$ & $P=5$ & $P=10$ \\
$k=3.1058$ & $k=2.8086$ & $k=2.4444$ & $k=9.8193$ & $k=8.7604$ & $k=7.4790$ \\
\hline 0 & 0.00 & 0.00 & 0.00 & 0.00 & 0.00 & 0.00 \\
1 & 0.80 & 0.82 & 0.85 & 0.82 & 0.84 & 0.87 \\
2 & 1.59 & 1.63 & 1.69 & 1.64 & 1.68 & 1.73 \\
3 & 2.38 & 2.45 & 2.54 & 2.46 & 2.52 & 2.59 \\
4 & 3.18 & 3.26 & 3.38 & 3.27 & 3.37 & 3.45 \\
5 & 3.97 & 4.09 & 4.23 & 4.09 & 4.19 & 4.31 \\
7 & 5.55 & 5.71 & 5.90 & 5.72 & 5.87 & 6.04 \\
10 & 7.93 & 8.16 & 8.46 & 8.16 & 8.38 & 8.63 \\
15 & 11.88 & 12.25 & 12.65 & 12.25 & 12.55 & 12.89 \\
20 & 15.81 & 16.28 & 16.85 & 16.27 & 16.70 & 17.22 \\
\hline
\end{tabular}


As one can easily see, $I(x)$ is slightly non linear in $x$. However it is a straightforward exercise to verify that if both individuals have the same coefficient of risk aversion, i.e. if $\alpha=\beta$, then we have from (9)

$$
I(x)=\frac{W_{2}+P}{W_{1}+W_{2}} x=\frac{100+P}{140} x
$$

In other words the indemnity $I(x)$ is then linear in $x$. Furthermore one notices that (10) can be obtained directly from (8).

Let us write the approximate value of $I(x)$ determined by (8) as

$$
I^{*}(x)=\gamma x
$$

Then Table III indicates the value of $\gamma$ for any $(P, \alpha, \beta)$. Comparing the approximate value $I^{*}(x)$ obtained from Table III with the exact value $I(x)$ given in Table II, one notices that $I^{*}(x)$ is an extremely good approximation for $I(x)$ for $x \leqslant 5$. The approximate value $I^{*}(x)$ overestimates slightly $I(x)$ for $5<x$ and the error then grows with $x$, though without ever becoming very significant.

Table III - Value of $\gamma$ according to $(P, \alpha, \beta)$

\begin{tabular}{|l|l|l|l|l|}
\hline \multicolumn{2}{|c|}{} & $P=1$ & $P=5$ & $P=10$ \\
\hline$\alpha=1 / 2$ & $\beta=1 / 2$ & .7214 & .7500 & .7857 \\
$\alpha=1 / 4$ & $\beta=1 / 2$ & .7953 & .8182 & .8462 \\
$\alpha=1 / 8$ & $\beta=1 / 2$ & .8182 & .8400 & .8652 \\
\hline
\end{tabular}

Table II indicates that for any $(P, \beta, x)$ the indemnity $I(x)$ grows when $\alpha$ diminishes. As an example, if $P=1, \beta=1 / 2$ and $x=5$ the indemnity should be equal to 3.97 for $\alpha=1 / 4$, compared with 4.09 for $\alpha=1 / 8$. In Appendix II it is indeed shown that $\partial I(x) / \partial \alpha$ is negative, a result which is easy to understand, as it will be seen. Since $R_{A 1}(x)=(1-\alpha) / x$, it follows that a decrease in $\alpha$ corresponds to an increase in the absolute risk aversion : ceteris paribus, an individual should prefer a larger indemnity as his absolute risk aversion grows.

As pointed out previously, the bargaining problem between the insurer and his client can be summarized in the choice of a premium $P$, since the indemnity scheme is then uniquely determined. Table II presents the indemnity scheme corresponding to three premium levels for two pairs $(\alpha, \beta)$. Of course, it is possible to derive an infinity of indemnity schemes from equation (9). However it is possible to illustrate the bargaining process from Table II despite its limitations.

We remember that both individuals are utility maximizers. Using the probability law of Table I one can compute the expected utility of each individual under each situation. These results are shown in Table IV. 
Table IV - Expected utility under each situation

\begin{tabular}{|c|c|c|c|c|c|c|}
\cline { 2 - 6 } & \multicolumn{3}{|c|}{$\alpha=1 / 4 \quad \beta=1 / 2$} & \multicolumn{3}{|c|}{$\alpha=1 / 8 \quad \beta=1 / 2$} \\
\cline { 2 - 7 } & \multicolumn{2}{|c|}{ expected utility } & $E[I(x)]$ & expected utility & \multirow{2}{*}{$E[I(x)]$} \\
\cline { 2 - 7 } & insured & insurer & & insured & insurer & \\
\hline $\begin{array}{l}\text { without } \\
\text { insurance }\end{array}$ & 2.44 & 10.00 & 0.00 & 1.56 & 10.00 & 0.00 \\
\hline $\mathbf{P}=1$ & 2.48 & 9.88 & 3.34 & 1.58 & 9.88 & 3.44 \\
\hline $\mathbf{P}=5$ & 2.42 & 10.08 & 3.43 & 1.56 & 10.07 & 3.53 \\
\hline $\mathbf{P}=10$ & 2.33 & 10.32 & 3.56 & 1.53 & 10.31 & 3.63 \\
\hline
\end{tabular}

Let us first study the case where $\alpha=1 / 4$ and $\beta=1 / 2$. Among the three available schemes, one sees that only the one where $P=1$ offers individual 1 (the potential insured) an expected utility which is larger than the expected utility attained when he takes no insurance. However the insurer would not be interested in accepting this particular scheme since his expected utility would then be equal to 9.88 , which is less than 10.00 the utility when he does not insure individual 1. If both individuals behave rationally they will prefer to continue without concluding a risk-sharing agreement rather than accepting one of the three schemes available. One notices that if $P=1$ then $E[I(x)]=3.34$, in accepting such a scheme the insurer would then be facing a negative expected gain.

Let us now turn our attention to the case where $\alpha=1 / 8$ and $\beta=1 / 2$. In this second case, individual 1 has a greater risk aversion. The figures presented in Table IV indicate that the only plan acceptable simultaneously by both parties is the one where $P=5$. By accepting this plan, individual 1 does not change his expected utility while the insurer sees his expected utility growing from 10.00 to 10.07 . One notices that $E[I(x)]=3.53$ which means that the insurer's expected gain is then equal to 1.47 . This latter figure can be interpretated as the risk premium that individual 1 is willing to pay to reduce the risk he is facing.

\section{The closed form solution for some specific utility functions}

As it has been observed while working out the numerical example of the previous section, it is not always possible to find a closed form solution to equation (5). However for three frequently used types of utility function it turns out that a closed form exists and that it corresponds precisely to the linear function which had been introduced as an approximation for $I(x)$ under (8).

\section{A. The quadratic}

Let $u_{1}(X)=a X+b X^{2}$ and $u_{2}(X)=c X+d X^{2}$ where the parameters are chosen such that $u_{i}^{\prime}(X)>0$ and $u_{i}^{\prime \prime}(X)<0$. Then solving (5) yields

$$
I(x)=\left[1+\frac{d\left[a+2 b\left(W_{1}-P\right)\right]}{b\left[c+2 d\left(W_{2}+P\right)\right]}\right] x
$$




\section{B. The exponential}

Let $u_{1}(X)=1-e^{-a X}$ and $u_{2}(X)=1-e^{-b X}$ where $a$ and $b$ are strictly positive. Then solving (5) yields

$$
I(x)=\frac{a}{a+b} x
$$

As it is often the case when using exponential utility functions, the solution is extremely simple. It is worth pointing out that the optimal indemnity scheme given by (12) is totally independent of the wealth of both individuals and of the premium. However in the bargaining process it is clear that these latter variables would play a determining role through the evaluation of the expected utility.

C. The logarithmic

Let $u_{1}(X)=a+b \log X$ and $u_{2}(X)=c+d \log X$ where $b$ and $d$ are strictly positive. Then solving (5) yields

$$
I(x)=\frac{W_{2}+P}{W_{1}+W_{2}} x
$$

One notices immediately that this solution corresponds exactly to the one obtained when using power utility functions with identical parameters (see (10)).

Comment. For the three types of utility function studied in this section, the optimal insurance scheme is therefore a coinsurance where the indemnity is a constant percentage of the loss.

\section{Conclusion}

This study has made it possible to illustrate the foundations of the economic theory of risk-sharing. One has seen that the concept of risk aversion is the basis for the existence of markets for the exchange of risk. The theory which has been presented can be subject to a few criticisms though.

For the sake of greater realism, the time dimension should eventually be introduced into the model. It would then be possible to illustrate how the insurance decision is related to the consumption decision. Furthermore greater realism would also ask for proper consideration of the transaction costs inherent to the problems studied.

\section{Appendix I}

We want to show that

Proof

$$
I(x) \approx \frac{R_{A 1}\left(W_{1}-P\right)}{R_{A 1}\left(W_{1}-P\right)+R_{A 2}\left(W_{2}+P\right)} x
$$

1. From (5) we have

$$
\frac{u_{2}^{\prime}\left(W_{2}+P-I\right)}{u_{1}^{\prime}\left(W_{1}-P-x+I\right)}=\frac{u_{2}^{\prime}\left(W_{2}+P\right)}{u_{1}^{\prime}\left(W_{1}-P\right)}
$$


2. According to the Taylor-Lagrange theorem there exists

$$
0<\Theta<1 \text { and } 0<\Delta<1
$$

such that (i) can be written

$$
\frac{u_{2}^{\prime}(W+P)-I u_{2}^{\prime \prime}\left(W_{2}+P-\Theta I\right)}{u_{1}^{\prime}\left(W_{1}-P\right)+(I-x) u_{1}^{\prime \prime}\left(W_{1}-P+\Delta(I-x)\right)}=\frac{u_{1}^{\prime}\left(W_{2}+P\right)}{u_{1}^{\prime}\left(W_{1}-P\right)}
$$

3. After some algebraic transformations (ii) yields

$$
I\left[\frac{u_{1}^{\prime \prime}\left(W_{1}-P\right) \cdot u_{2}^{\prime \prime}\left(W_{2}+P-\Theta I\right)}{u_{1}^{\prime \prime}\left(W_{1}-P+\Delta(I-x)\right) \cdot u_{2}^{\prime \prime}\left(W_{2}+P\right)} \frac{R_{A 2}\left(W_{2}+P\right)}{R_{A 1}\left(W_{1}-P\right)}+1\right\rceil=x
$$

and (8) follows.

Q.E.D.

\section{Appendix II}

If $u_{1}(X)=X^{\alpha}, u_{2}(X)=X^{\beta}, 0<\alpha<1$ and $0<\beta<1$

then

$$
\frac{\partial I(x)}{\partial \alpha}<0
$$

\section{Proof}

1. Differentiating in (9) with respect to $\alpha$ yields

$$
\frac{\partial I(x)}{\partial \alpha}=-\frac{\left[\frac{40-P-x+I(x)}{40-P}\right] \log \left[\frac{40-P-x+I(x)}{40-P}\right]}{(\alpha-1)\left[\frac{40-P-x+I(x)}{40-P}\right]^{\alpha-2}+(\beta-1)\left[\frac{100+P-I(X)}{100-P}\right]^{\beta-2}}
$$

2. We know that

$$
\begin{aligned}
& x>0 \Rightarrow I(x)<x \\
& P+x-I(x)>P
\end{aligned}
$$

therefore

and

$$
\log \left[\frac{40-P-x+I(x)}{40-P}\right]<0
$$

3. It follows that $\frac{\partial I(x)}{\partial \alpha}<0$.

Q.E.D.

\section{REFERENCES}

1. ARROW, Kenneth: "Uncertainty and the welfare economics of medical care ", American Economic Review, Vol. 53, 1963, 941-973.

2. BORCH, Karl: “The safety loading of reinsurance premiums", Skandinavisk Aktuarietidskrift, 1960, 163-184. 